Mathematical Research Letters 7, 13-24 (2000)

\title{
PERVERSE SHEAVES ON AFFINE GRASSMANNIANS AND LANGLANDS DUALITY
}

\author{
IVAN MiRKOVIĆ AND KARI VILONEN
}

\section{Introduction}

In this paper we outline a proof of a geometric version of the Satake isomorphism. Namely, given a connected, complex algebraic reductive group $G$ we show that the tensor category of representations of the dual group ${ }^{L} G$ is naturally equivalent to a certain category of perverse sheaves on the affine Grassmannian of $G$. This can be extended to give a topological realization of algebraic representations of ${ }^{L} G$ over any commutative ring $\mathbb{k}$ - the category of $\mathbb{k}$-representations of ${ }^{L} G$ is equivalent to a category of perverse sheaves on the affine Grassmannian (a complex algebraic variety), with coefficients in $\mathbb{k}$.

The above result over the complex numbers is not new. This case has been treated by Ginzburg in [Gi] and some of the arguments in section 5 of this paper are borrowed from [Gi]. However, at crucial points our proof differs from Ginzburg's. First, we use a more "natural" commutativity constraint for the convolution product. This commutativity constraint, explained in section 3 , is due to Drinfeld and was explained to us by Beilinson. Secondly, in section 4, we give a direct proof that the global cohomology functor is exact and decompose this cohomology functor into a direct sum of weights (Theorem 4.3). The geometry underlying our arguments leads to a construction of a canonical basis of Weyl modules given by algebraic cycles. Another consequence is an explicit construction of the group algebra of ${ }^{L} G$ in terms of the affine Grassmannian. We completely avoid the use of the decomposition theorem of [BBD] which makes our techniques applicable to perverse sheaves with coefficients over an arbitrary commutative ring $\mathbb{k}$. In sections $1-5$ we state the results when $\mathbb{k}$ is a field of characteristic zero. The modifications needed for the general case are in $\S 6$. In $\S 7$ we derive the classical Satake isomorphism, for this we switch the setting to the affine Grassmannian defined over a finite field and $\ell$-adic perverse sheaves. This note contains indications of proofs of some of the results. The details will appear elsewhere.

Received November 2, 1999.

I. Mirković was partially supported by NSF

K.Vilonen was partially supported by NSA and NSF 


\section{The convolution product}

Let $G$ be a connected, complex algebraic reductive group. Denote by $\mathcal{O}=\mathbb{C}[[t]]$ the ring of formal power series in one variable and by $\mathcal{K}=\mathbb{C}((t))$ its fraction field, the field of formal Laurent series. The affine Grassmannian, as a set, is defined as $\mathcal{G}=G(\mathcal{K}) / G(\mathcal{O})$, where, as usual, $G(\mathcal{K})$ and $G(\mathcal{O})$ denote the sets of the $\mathcal{K}$-valued and the $\mathcal{O}$-valued points of $G$ respectively. The sets $G(\mathcal{K}), G(\mathcal{O})$, and $\mathcal{G}$ have an algebraic structure as $\mathbb{C}$-spaces. The space $G(\mathcal{O})$ is a group scheme over $\mathbb{C}$ but the spaces $G(\mathcal{K})$ and $\mathcal{G}$ are only ind-schemes ${ }^{1}$. To see that $G(\mathcal{K})$ is an ind-scheme, one embeds $G$ in $S L_{N}(\mathbb{C})$. The filtration by order of pole in $S L_{N}(\mathcal{K})$ induces a filtration of $G(\mathcal{K})$ which exhibits $G(\mathcal{K})$ as an inductive limit of schemes. The filtration above is invariant under the (right) action of $G(\mathcal{O})$ on $G(\mathcal{K})$ and thus, after taking the quotient of $G(\mathcal{K})$ by $G(\mathcal{O})$ one gets a filtration of $\mathcal{G}$ which exhibits it as a union of finite dimensional projective schemes. Furthermore, the morphism $\pi: G(\mathcal{K}) \rightarrow \mathcal{G}$ is locally trivial in the Zariski topology, i.e., there exists a Zariski open subset $U \subset \mathcal{G}$ such that $\pi^{-1}(U) \cong U \times G(\mathcal{O})$ and $\pi$ restricted to $U \times G(\mathcal{O})$ is simply projection to the first factor. For details see for example [BL1,LS].

The group scheme $G(\mathcal{O})$ acts on $\mathcal{G}$ with finite dimensional orbits. In order to describe the orbit structure, let us fix a maximal torus $T \subset G$. We write $W$ for the Weyl group and $X_{*}(T)$ for the coweights $\operatorname{Hom}\left(\mathbb{C}^{*}, T\right)$. Then the $G(\mathcal{O})$ orbits on $\mathcal{G}$ are parameterized by the $W$-orbits in $X_{*}(T)$, and given $\lambda \in X_{*}(T)$ the $G(\mathcal{O})$-orbit associated to it is $\mathcal{G}_{\lambda}=G(\mathcal{O}) \cdot \lambda \subset \mathcal{G}$, where we have identified $X_{*}(T)$ as a subset of $G(\mathcal{K})$.

Let $\mathbb{k}$ be a field of characteristic zero, which we fix for the sections 1-5. All sheaves that we encounter in this paper will be sheaves in the classical topology with the exception of $\S 7$. We denote by $\mathrm{P}_{G(\mathcal{O})}(\mathcal{G}, \mathbb{k})$ the category of $G(\mathcal{O})$-equivariant perverse $\mathbb{k}$-sheaves on $\mathcal{G}$ with finite dimensional support and by $\mathrm{P}_{\mathcal{S}}(\mathcal{G}, \mathbb{k})$ the category of perverse $\mathbb{k}$-sheaves on $\mathcal{G}$ which are constructible with respect to the orbit stratification $\mathcal{S}$ of $\mathcal{G}$ and which have finite dimensional support. We use the notational conventions of $[\mathrm{BBD}]$ for perverse sheaves, in particular, in order for the constant sheaf on a $G(\mathcal{O})$-orbit $\mathcal{G}_{\lambda}$ to be perverse it has to be placed in degree $-\operatorname{dim} \mathcal{G}_{\lambda}$.

Proposition 2.1. The forgetful functor $\mathrm{P}_{G(\mathcal{O})}(\mathcal{G}, \mathbb{k}) \rightarrow \mathrm{P}_{\mathcal{S}}(\mathcal{G}, \mathbb{k})$ is an equivalence of categories.

We will now put a tensor category structure on $\mathrm{P}_{G(\mathcal{O})}(\mathcal{G}, \mathbb{k})$ via the convolution product. Consider the following diagram of maps (of sets)

$$
\mathcal{G} \times \mathcal{G} \stackrel{p}{\leftarrow} G(\mathcal{K}) \times \mathcal{G} \stackrel{q}{\longrightarrow} G(\mathcal{K}) \times_{G(\mathcal{O})} \mathcal{G} \stackrel{m}{\longrightarrow} \mathcal{G} .
$$

Here $G(\mathcal{K}) \times_{G(\mathcal{O})} \mathcal{G}$ denotes the quotient of $G(\mathcal{K}) \times \mathcal{G}$ by $G(\mathcal{O})$ where the action is given on the $G(\mathcal{K})$-factor via right multiplication by an inverse and on the

\footnotetext{
${ }^{1} \mathrm{By}$ an ind-scheme we mean an ind-scheme in a strict sense, i.e., an inductive system of schemes where all maps are closed embeddings.
} 
$\mathcal{G}$-factor by left multiplication. The $p$ and $q$ are projection maps and $m$ is the multiplication map. All other terms in (2.2) have been given a structure of an ind-scheme except $G(\mathcal{K}) \times_{G(\mathcal{O})} \mathcal{G}$. The description of this structure is easier in the global context of section 3 where it is a special case of a more general construction and thus we postpone the details. We define the convolution product $A_{1} * A_{2}$ of $A_{1}, A_{2} \in \mathrm{P}_{G(\mathcal{O})}(\mathcal{G}, \mathbb{k})$ by the formula

$$
A_{1} * A_{2}=R m_{*} \tilde{A} \quad \text { where } q^{*} \tilde{A}=p^{*}\left(A_{1} \otimes A_{2}\right) .
$$

To make sense of this definition we first use the fact that $p$ and $q$ are locally trivial in the Zariski topology. This guarantees the existence of $\tilde{A} \in \mathrm{P}_{G(\mathcal{O})}\left(G(\mathcal{K}) \times_{G(\mathcal{O})}\right.$ $\mathcal{G}, \mathbb{k})$. To see the local triviality of $q$ one can use the same arguments as for example in [BL1,LS], and as was pointed out above, the local triviality of $p$ is proved in those references. It remains to show that $R m_{*} \tilde{A} \in \mathrm{P}_{G(\mathcal{O})}(\mathcal{G}, \mathbb{k})$. To that end we introduce the notion of a stratified semi-small map.

Let us consider two complex stratified spaces $(Y, \mathcal{T})$ and $(X, \mathcal{S})$ and a map $f: Y \rightarrow X$. We assume that the two stratifications are locally trivial with connected strata and that $f$ is a stratified with respect to the stratifications $\mathcal{T}$ and $\mathcal{S}$, i.e., that for any $T \in \mathcal{T}$ the image $f(T)$ is a union of strata in $\mathcal{S}$ and for any $S \in \mathcal{S}$ the map $f \mid f^{-1}(S): f^{-1}(S) \rightarrow S$ is locally trivial in the stratified sense. We say that $f$ is a stratified semi-small map if

a) for any $T \in \mathcal{T}$ the map $f \mid \bar{T}$ is proper,

b) for any $T \in \mathcal{T}$ and any $S \in \mathcal{S}$ such that $S \subset f(\bar{T})$ we have

$$
\operatorname{dim}\left(f^{-1}(x) \cap \bar{T}\right) \leq \frac{1}{2}(\operatorname{dim} f(\bar{T})-\operatorname{dim} S),
$$

for any (and thus all) $x \in S$.

Next the notion of a small stratified map. We say that $f$ is a small stratified map if there exists a (non-trivial) open stratified subset $W$ of $Y$ such that

a) for any $T \in \mathcal{T}$ the map $f \mid \bar{T}$ is proper,

b) the map $f \mid W: W \rightarrow f(W)$ is proper and has finite fibers,

c) for any $T \in \mathcal{T}, T \subset W$, and any $S \in \mathcal{S}$ such that $S \subset f(\bar{T})-f(T)$ we have

$$
\operatorname{dim}\left(f^{-1}(x) \cap \bar{T}\right) \leq \frac{1}{2}(\operatorname{dim} f(\bar{T})-\operatorname{dim} S),
$$

for any (and thus all) $x \in S$.

The result below follows directly from dimension counting:

Lemma 2.6. If $f$ is a semismall stratified map then $R f_{*} A \in \mathrm{P}_{\mathcal{S}}(X, \mathbb{k})$ for all $A \in \mathrm{P}_{\mathcal{T}}(Y, \mathbb{k})$. If $f$ is a small stratified map then, with any $W$ as above, and any $A \in \mathrm{P}_{\mathcal{T}}(W, \mathbb{k})$, we have $R f_{*} j_{!_{*}} A=\tilde{j}_{!_{*}} f_{*} A$, where $j: W \hookrightarrow Y$ and $\tilde{j}: f(W) \hookrightarrow X$ denote the two inclusions.

We apply the above considerations, in the semismall case, to our situation. We take $Y=G(\mathcal{K}) \times_{G(\mathcal{O})} \mathcal{G}$ and choose $\mathcal{T}$ to be the stratification whose strata are 
$p^{-1}\left(\mathcal{G}_{\lambda}\right) \times_{G(\mathcal{O})} \mathcal{G}_{\mu}$, for $\lambda, \mu \in X_{*}(T)$. We also let $X=\mathcal{G}, \mathcal{S}$ the stratification by $G(\mathcal{O})$-orbits, and choose $f=m$. To conclude the construction of the convolution product on $\mathrm{P}_{G(\mathcal{O})}(\mathcal{G}, \mathbb{k})$ it suffices to note that the sheaf $\tilde{A}$ is constructible with respect to the stratification $\mathcal{T}$ and appeal to the following

Theorem 2.7. The multiplication map $G(\mathcal{K}) \times_{G(\mathcal{O})} \mathcal{G} \stackrel{m}{\longrightarrow} \mathcal{G}$ is a stratified semismall map with respect to the stratifications above.

For an outline of proof, see remark 4.11.

One can define the convolution product of three sheaves completely analogously to (2.3). This gives an associativity constraint for the convolution product thus giving $\mathrm{P}_{G(\mathcal{O})}(\mathcal{G}, \mathbb{k})$ the structure of an associative tensor category. In the next section we construct a commutativity constraint for the convolution product.

\section{The commutativity constraint}

In order to construct the commutativity constraint we will need to consider the convolution product in the global situation. Let $X$ be a smooth curve over the complex numbers. Let $x \in X$ be a closed point and denote by $\mathcal{O}_{x}$ the completion of the local ring at $x$ and by $\mathcal{K}_{x}$ its fraction field. Then the Grassmannian $\mathcal{G}_{x}=G\left(\mathcal{K}_{x}\right) / G\left(\mathcal{O}_{x}\right)$ represents the following functor from $\mathbb{C}$-algebras to sets :

$R \mapsto\left\{\mathcal{F}\right.$ a $G$-torsor on $X_{R}, \nu: G \times X_{R}^{*} \rightarrow \mathcal{F} \mid X_{R}^{*}$ a trivialization on $\left.X_{R}^{*}\right\}$.

Here the pairs $(\mathcal{F}, \nu)$ are to be taken up to isomorphism, $X_{R}=X \times \operatorname{Spec}(R)$, and $X_{R}^{*}=(X-\{x\}) \times \operatorname{Spec}(R)$. For details see for example [BL1,BL2,LS]. We now globalize this construction and at the same time form the Grassmannian at several points on the curve. Denote the $n$ fold product by $X^{n}=X \times \cdots \times X$ and consider the functor

$$
R \mapsto\left\{\begin{array}{l}
\left(x_{1}, \ldots, x_{n}\right) \in X^{n}(R), \quad \mathcal{F} \text { a } G \text {-torsor on } X_{R}, \\
\nu_{\left(x_{1}, \ldots, x_{n}\right)} \text { a trivialization of } \mathcal{F} \text { on } X_{R}-\cup x_{i}
\end{array}\right\} .
$$

Here we think of the points $x_{i}: \operatorname{Spec}(R) \rightarrow X$ as subschemes of $X_{R}$ by taking their graphs. One sees that the functor in (3.2) is represented by an ind-scheme $\mathcal{G}_{X}^{(n)}$. Of course $\mathcal{G}_{X}^{(n)}$ is an ind-scheme over $X^{n}$ and its fiber over the point $\left(x_{1}, \ldots, x_{n}\right)$ is simply $\prod_{i=1}^{k} \mathcal{G}_{y_{i}}$, where $\left\{y_{1}, \ldots, y_{k}\right\}=\left\{x_{1}, \ldots, x_{n}\right\}$, with all the $y_{i}$ distinct. We write $\mathcal{G}_{X}^{(1)}=\mathcal{G}_{X}$.

We will now extend the diagram of maps (2.2), which was used to define the convolution product, to the global situation, i.e., to a diagram of ind-schemes over $X^{2}$ :

$$
\mathcal{G}_{X} \times \mathcal{G}_{X} \stackrel{p}{\longleftarrow}{\widetilde{\mathcal{G}_{X} \times \mathcal{G}_{X}}}_{\stackrel{q}{\longrightarrow}}^{\rightarrow} \mathcal{G}_{X} \tilde{\times} \mathcal{G}_{X} \stackrel{m}{\longrightarrow} \mathcal{G}_{X}^{(2)} .
$$

Roughly, the diagram starts with a pair of torsors, each trivialized off one point. One chooses a trivialization of the first torsor near the second point, and uses it to glue the torsors. 
More precisely, $\widetilde{\mathcal{G}_{X} \times \mathcal{G}_{X}}$ denotes the ind-scheme representing the functor

$$
R \mapsto\left\{\begin{array}{l}
\left(x_{1}, x_{2}\right) \in X^{2}(R) ; \mathcal{F}_{1}, \mathcal{F}_{2} G \text {-torsors on } X_{R} ; \\
\nu_{i} \text { a trivialization of } \mathcal{F}_{i} \text { on } X_{R}-x_{i}, \text { for } i=1,2 ; \\
\mu_{1} \text { a trivialization of } \mathcal{F}_{1} \text { on } \widehat{\left(X_{R}\right)}
\end{array}\right\}
$$

where ${\widehat{\left(X_{R}\right)}}_{x_{2}}$ denotes the formal neighborhood of $x_{2}$ in $X_{R}$. The "twisted product" $\mathcal{G}_{X} \tilde{\times} \mathcal{G}_{X}$ is the ind-scheme representing the functor

$$
R \mapsto\left\{\begin{array}{l}
\left(x_{1}, x_{2}\right) \in X^{2}(R) ; \mathcal{F}_{1}, \mathcal{F} G \text {-torsors on } X_{R} ; \nu_{1} \text { a trivialization } \\
\text { of } \mathcal{F}_{1} \text { on } X_{R}-x_{1} ; \eta: \mathcal{F}_{1}\left|\left(X_{R}-x_{2}\right) \stackrel{\simeq}{\longrightarrow}\right|\left(X_{R}-x_{2}\right)
\end{array}\right\}
$$

It remains to describe the morphisms $p, q$, and $m$ in (3.3). Because all the spaces in (3.3) are ind-schemes over $X^{2}$, and all the functors involve the choice of the same $\left(x_{1}, x_{2}\right) \in X^{2}(R)$ we omit it in the formulas below. The morphism $p$ simply forgets the choice of $\mu_{1}$, the morphism $q$ is given by the natural transformation

$$
\left(\mathcal{F}_{1}, \nu_{1}, \mu_{1} ; \mathcal{F}_{2}, \nu_{2}\right) \mapsto\left(\mathcal{F}_{1}, \nu_{1}, \mathcal{F}, \eta\right)
$$

where $\mathcal{F}$ is the $G$-torsor gotten by gluing $\mathcal{F}_{1}$ on $X_{R}-x_{2}$ and $\mathcal{F}_{2}$ on $\widehat{\left(X_{R}\right)} x_{x_{2}}$ using the isomorphism induced by $\nu_{2} \circ \mu_{1}^{-1}$ between $\mathcal{F}_{1}$ and $\mathcal{F}_{2}$ on $\left(X_{R}-x_{2}\right) \cap \widehat{\left(X_{R}\right)} x_{2}$. The morphism $m$ is given by the natural transformation

$$
\left(\mathcal{F}_{1}, \nu_{1}, \mathcal{F}, \eta\right) \mapsto(\mathcal{F}, \nu)
$$

where $\nu=\left(\eta \circ \nu_{1}\right) \mid\left(X_{R}-x_{1}-x_{2}\right)$.

Next, the global analog of $G(\mathcal{O})$ is the group-scheme $G_{X}^{(n)}(\mathcal{O})$ which represents the functor

$$
R \mapsto\left\{\begin{array}{l}
\left(x_{1}, \ldots, x_{n}\right) \in X^{n}(R), \mathcal{F} \text { the trivial } G \text {-torsor on } X_{R}, \\
\mu_{\left(x_{1}, \ldots, x_{n}\right)} \text { a trivialization of } \mathcal{F} \text { on } \widehat{\left(X_{R}\right)}\left(x_{1} \cup \ldots \cup x_{n}\right)
\end{array}\right\} .
$$

Just as in section 2, we define the convolution product of $\mathcal{B}_{1}, \mathcal{B}_{2} \in$ $\mathcal{P}_{G_{X}(\mathcal{O})}\left(\mathcal{G}_{X}, \mathbb{k}\right)$ by the formula

$$
\mathcal{B}_{1}^{*}{ }_{X}^{*} \mathcal{B}_{2}=R m_{*} \tilde{\mathcal{B}}, \quad \text { where } q^{*} \tilde{\mathcal{B}}=p^{*}\left(\mathcal{B}_{1} \otimes \mathcal{B}_{2}\right) .
$$

Precisely as in section 2 , the sheaf $\tilde{\mathcal{B}}$ exists because $q$ is locally, even in the Zariski topology, a product. Furthermore, the map $m$ is a stratified small map - regardless of the stratification on $X$. To see this, let us denote by $\Delta \subset X^{2}$ the diagonal and set $U=X^{2}-\Delta$. Then we can take, in definition (2.5), as $W$ the locus of points lying over $U$. That $m$ is small now follows as $m$ is an 
isomorphism over $U$ and over points of $\Delta$ the map $m$ coincides with its analogue in section 2 which is semi-small by theorem 2.7 .

Let us now, for simplicity, choose $X=\mathbb{A}^{1}$. Then the choice of a global coordinate on $\mathbb{A}^{1}$, trivializes $\mathcal{G}_{X}$ over $X$; let us write $\rho: \mathcal{G}_{X} \rightarrow \mathcal{G}$ for the projection. Let us denote $\rho^{0}=\rho^{*}[1]: \mathcal{P}_{G(\mathcal{O})}(\mathcal{G}, \mathbb{k}) \rightarrow \mathcal{P}_{G_{X}(\mathcal{O})}\left(\mathcal{G}_{X}, \mathbb{k}\right)$. By restricting $\mathcal{G}_{X}^{(2)}$ to the diagonal $\Delta \cong X$ and to $U$, and observing that these restrictions are isomorphic to $\mathcal{G}_{X}$ and to $\left(\mathcal{G}_{X} \times \mathcal{G}_{X}\right) \mid U$ respectively, we get the following diagram

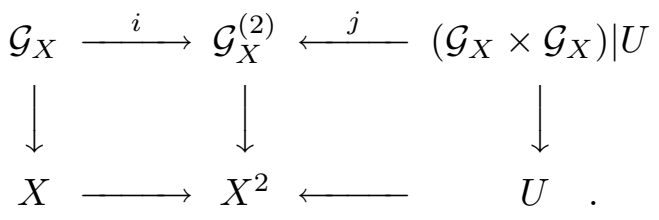

Lemma 3.11. For $\mathcal{A}_{1}, \mathcal{A}_{2} \in \mathcal{P}_{G(\mathcal{O})}(\mathcal{G}, \mathbb{k})$ we have

a) $\rho^{0} \mathcal{A}_{1} \underset{X}{*} \rho^{0} \mathcal{A}_{2} \cong j ! *\left(\left(\rho^{0} \mathcal{A}_{1} \otimes \rho^{0} \mathcal{A}_{2}\right) \mid U\right)$,

b) $\rho^{0}\left(\mathcal{A}_{1} * \mathcal{A}_{2}\right) \cong i^{0}\left(\rho^{0} \mathcal{A}_{1} \underset{X}{*} \rho^{0} \mathcal{A}_{2}\right)$.

Part a) of the lemma follows from smallness of $m$ and lemma 2.6.

Lemma 3.11 gives us the following sequence of isomorphisms:

$$
\begin{aligned}
\rho^{0}\left(\mathcal{A}_{1} * \mathcal{A}_{2}\right) & \cong i^{0} j_{! *}\left(\left(\rho^{0} \mathcal{A}_{1} \otimes \rho^{0} \mathcal{A}_{2}\right) \mid U\right) \\
& \cong i^{*} j_{! *}\left(\left(\rho^{0} \mathcal{A}_{2} \otimes \rho^{0} \mathcal{A}_{1}\right) \mid U\right) \cong \rho^{0}\left(\mathcal{A}_{2} * \mathcal{A}_{1}\right)
\end{aligned}
$$

Specializing this isomorphism to (any) point on the diagonal yields a functorial isomorphism between $\mathcal{A}_{1} * \mathcal{A}_{2}$ and $\mathcal{A}_{2} * \mathcal{A}_{1}$. This gives us a commutativity constraint making $\mathcal{P}_{G(\mathcal{O})}(\mathcal{G}, \mathbb{k})$ into a tensor category.

Remark 3.13. The construction of the commutativity constraint can be carried out in a more elegant way as follows. We first observe that the image of the embedding $\rho^{0}=\rho^{*}[1]: \mathcal{P}_{G(\mathcal{O})}(\mathcal{G}, \mathbb{k}) \rightarrow \mathcal{P}_{G_{X}(\mathcal{O})}\left(\mathcal{G}_{X}, \mathbb{k}\right)$ consists precisely of objects in $\mathcal{P}_{G_{X}(\mathcal{O})}\left(\mathcal{G}_{X}, \mathbb{k}\right)$ which are "constant" along $X$. This subcategory of $\mathcal{P}_{G_{X}(\mathcal{O})}\left(\mathcal{G}_{X}, \mathbb{k}\right)$ coincides with $\mathcal{P}_{\tilde{G}_{X}(\mathcal{O})}\left(\mathcal{G}_{X}, \mathbb{k}\right)$, where $\tilde{G}_{X}(\mathcal{O})$ denotes the semi direct product of $G_{X}(\mathcal{O})$ and the groupoid which consists of pairs of points $(x, y) \in X \times X$ together with an isomorphism between the formal neighborhood of $x$ and the formal neighborhood of $y$. Now $\rho^{0}=\rho^{*}[1]$ : $\mathcal{P}_{G(\mathcal{O})}(\mathcal{G}, \mathbb{k}) \rightarrow \mathcal{P}_{\tilde{G}_{X}(\mathcal{O})}\left(\mathcal{G}_{X}, \mathbb{k}\right)$ is an equivalence whose inverse is $i^{0}=i^{*}[-1]$, where $i: \mathcal{G}_{x} \hookrightarrow \mathcal{G}_{X}$ is the inclusion. If $X$ is an arbitrary smooth curve then the functor $i^{0}: \mathcal{P}_{\tilde{G}_{X}(\mathcal{O})}\left(\mathcal{G}_{X}, \mathbb{k}\right) \rightarrow \mathcal{P}_{G(\mathcal{O})}(\mathcal{G}, \mathbb{k})$ still has meaning and is an equivalence of categories. It is clear that the convolution product (3.9) gives us a convolution product on the category $\mathcal{P}_{\tilde{G}_{X}(\mathcal{O})}\left(\mathcal{G}_{X}, \mathbb{k}\right)$. Thus, we can give the construction of the commutativity constraint in terms of $\mathcal{P}_{\tilde{G}_{X}(\mathcal{O})}\left(\mathcal{G}_{X}, \mathbb{k}\right)$ and $i^{0}$ without specializing to $X=\mathbb{A}^{1}$ and choosing a global coordinate. 


\section{The fiber functor}

Let $\operatorname{Vec}_{\mathbb{k}}^{\epsilon}$ denote the category of finite dimensional $\mathbb{Z} / 2 \mathbb{Z}$ - graded (super) vector spaces over $\mathbb{k}$. Let us consider the global cohomology functor as $\mathbb{H}^{*}$ : $\mathrm{P}_{G(\mathcal{O})}(\mathcal{G}, \mathbb{k}) \rightarrow \operatorname{Vec}_{\mathbb{k}}^{\epsilon}$, where we only keep track of the parity of the grading on global cohomology. Then:

(4.1) The functor $\mathbb{H}^{*}: \mathrm{P}_{G(\mathcal{O})}(\mathcal{G}, \mathbb{k}) \rightarrow \operatorname{Vec}_{\mathbb{k}}^{\epsilon}$ is a tensor functor with respect to the commutativity constraint of section 3 .

Writing $r$ for the map $r: \mathcal{G}_{X}^{(2)} \rightarrow X^{2}$, this statement is an immediate consequence of :
a) $R r_{*}\left(\rho^{0}\left(A_{1}\right) *_{X} \rho^{0}\left(A_{2}\right)\right) \mid U$ is the constant sheaf $\mathbb{H}^{*}\left(A_{1}\right) \otimes \mathbb{H}^{*}\left(A_{2}\right)$,
b) $R r_{*}\left(\rho^{0}\left(A_{1}\right) *_{X} \rho^{0}\left(A_{2}\right)\right) \mid \Delta=\rho^{0}\left(\mathbb{H}^{*}\left(A_{1} * A_{2}\right)\right)$,
c) $R r_{*}\left(\rho^{0}\left(A_{1}\right) *_{X} \rho^{0}\left(A_{2}\right)\right)$ is a constant sheaf.

The claims a) and b) follow from lemma 3.11. It remains to note that, in the notation of formula (3.9), the sheaf $R(r \circ m)_{*} \tilde{B}$ is constant; this implies c)

Let $\mathrm{Vec}_{\mathbb{k}}$ denote the category of finite dimensional vector spaces over $\mathbb{k}$. To make $\mathbb{H}^{*}: \mathrm{P}_{G(\mathcal{O})}(\mathcal{G}, \mathbb{k}) \rightarrow \mathrm{Vec}_{\mathbb{k}}$ into a tensor functor we alter, following Beilinson and Drinfeld, the commutativity constraint of $\S 3$ slightly. We consider the constraint from $\S 3$ on the category $\mathrm{P}_{G(\mathcal{O})}(\mathcal{G}, \mathbb{k}) \otimes \operatorname{Vec}_{\mathbb{k}}^{\epsilon}$ and restrict it to a subcategory that we identify with $\mathrm{P}_{G(\mathcal{O})}(\mathcal{G}, \mathbb{k})$. Divide $\mathcal{G}$ into unions of connected components $\mathcal{G}=\mathcal{G}_{+} \cup \mathcal{G}_{-}$so that the dimension of $G(\mathcal{O})$-orbits is even in $\mathcal{G}_{+}$ and odd in $\mathcal{G}_{-}$. This gives a $\mathbb{Z}_{2}$-grading on the category $\mathrm{P}_{G(\mathcal{O})}(\mathcal{G}, \mathbb{k})$ hence a new $\mathbb{Z}_{2}$-grading on $\mathrm{P}_{G(\mathcal{O})}(\mathcal{G}, \mathbb{k}) \otimes \operatorname{Vec}_{\mathbb{k}}^{\epsilon}$. The subcategory of even objects is identified with $\mathrm{P}_{G(\mathcal{O})}(\mathcal{G}, \mathbb{k})$ by forgetting the grading.

4.3 Proposition. The functor $\mathbb{H}^{*}: \mathrm{P}_{G(\mathcal{O})}(\mathcal{G}, \mathbb{k}) \rightarrow \mathrm{Vec}_{\mathbb{k}}$ is a tensor functor with respect to the above commutativity constraint.

We now come to the main technical result of this paper. In order to state it we will fix some further notation. We choose a Borel subgroup $B \subset G$ which contains the maximal torus $T$. This, of course, determines a choice of positive roots. Let $N$ denote the unipotent radical of $B$. As usual, we denote by $\rho$ half the sum of positive roots of $G$. For any $\nu \in X_{*}(T)$ we write ht $(\nu)$ for the height of $\nu$ with respect to $\rho$. The $N(\mathcal{K})$-orbits on $\mathcal{G}$ are parameterized by $X_{*}(T)$; to each $\nu \in X_{*}(T)=\operatorname{Hom}\left(\mathbb{C}^{*}, T\right)$ we associate the $N(\mathcal{K})$-orbit $S_{\nu} \stackrel{\text { def }}{=} N(\mathcal{K}) \cdot \nu$. Note that these orbits are neither of finite dimension nor of finite codimension.

\section{Theorem 4.4.}

a) For all $\mathcal{A} \in \mathrm{P}_{G(\mathcal{O})}(\mathcal{G}, \mathbb{k})$ we have

$$
\mathrm{H}_{c}^{k}\left(S_{\nu}, \mathcal{A}\right)=0 \quad \text { if } k \neq 2 \mathrm{ht}(\nu) .
$$

In particular, the functors $\mathrm{H}_{c}^{2}{ }^{\mathrm{ht}(\nu)}\left(S_{\nu}, \quad\right): \mathrm{P}_{G(\mathcal{O})}(\mathcal{G}, \mathbb{k}) \rightarrow \mathrm{Vec}_{\mathbb{k}}$ are exact. 
b) We have a natural equivalence of functors

$$
\mathbb{H}^{*} \cong \bigoplus_{\nu \in X_{*}(T)} \mathrm{H}_{c}^{2 \mathrm{ht}(\nu)}\left(S_{\nu},\right): \mathrm{P}_{G(\mathcal{O})}(\mathcal{G}, \mathbb{k}) \rightarrow \operatorname{Vec}_{\mathbb{k}}
$$

This result immediately gives the following consequence:

Corollary 4.5. The global cohomology functor $\mathbb{H}^{*}: \mathrm{P}_{G(\mathcal{O})}(\mathcal{G}, \mathbb{k}) \rightarrow \operatorname{Vec}_{\mathbb{k}}$ is exact.

Here is a brief outline of the proof of theorem 4.4. Let us consider unipotent radical $\bar{N}$ of the Borel $\bar{B}$ opposite to $B$. The $\bar{N}(\mathcal{K})$-orbits on $\mathcal{G}$ are parameterized by $X_{*}(T)$ : to each $\nu \in X_{*}(T)$ we associate the orbit $T_{\nu}=\bar{N}(\mathcal{K}) \cdot \nu$. Recall that the $G(\mathcal{O})$-orbits are parameterized by $X_{*}(T) / W$. The orbit $S_{\nu}$ meets $\mathcal{G}_{\lambda}$ iff $\nu \in \overline{\mathcal{G}_{\lambda}} \cap X_{*}(T)$, then

a) $\operatorname{dim}\left(S_{\nu} \cap \mathcal{G}_{\lambda}\right)=\operatorname{ht}(\nu+\lambda)$, if $\lambda$ is chosen dominant,

b) $\operatorname{dim}\left(T_{\nu} \cap \mathcal{G}_{\lambda}\right)=-\operatorname{ht}(\nu+\lambda)$, if $\lambda$ is chosen anti-dominant,

c) the intersections in a) and b) are of pure dimension.

In proving estimates a) - c) we use the fact that the boundary $\partial S_{\nu}$ is given by one equation in the closure $\bar{S}_{\nu}$. From the dimension estimates $(4.6 \mathrm{a}, \mathrm{b})$ above we conclude immediately that

$$
\begin{array}{ll}
\mathrm{H}_{c}^{k}\left(S_{\nu}, \mathcal{A}\right)=0 & \text { if } k>2 \mathrm{ht}(\nu), \\
\mathrm{H}_{T_{\nu}}^{k}(\mathcal{G}, \mathcal{A})=0 & \text { if } k<2 \operatorname{ht}(\nu) .
\end{array}
$$

Theorem 4.4 follows immediately from (4.7) and the following statement:

$$
\mathrm{H}_{c}^{k}\left(S_{\nu}, \mathcal{A}\right)=\mathrm{H}_{T_{\nu}}^{k}(\mathcal{G}, \mathcal{A}), \quad \text { for all } k
$$

To see (4.8) we use the fact that $N(\mathcal{K})$-orbits and $\bar{N}(\mathcal{K})$-orbits are in general position with respect to each other.

Remark 4.9. The decomposition of functors in theorem 4.4b is independent of the choice of $N$. In the case of $N$ and its opposite unipotent subgroup $\bar{N}$ the corresponding decompositions are explicitly related by $\mathrm{H}_{S_{\nu}}^{k}(\mathcal{G}, \mathcal{A}) \cong \mathrm{H}_{T_{w_{0} \cdot \nu}}^{k}(\mathcal{G}, \mathcal{A})$, where $w_{0}$ is the longest element in the Weyl group. From this, and (4.8), we conclude that we could state theorem 4.4 replacing the functors $\mathrm{H}_{c}^{2 \mathrm{ht}(\nu)}\left(S_{\nu}, \quad\right)$ by the equivalent set of functors $\mathrm{H}_{S_{\nu}}^{2 \mathrm{ht}(\nu)}(\mathcal{G}, \quad)$, where $\mathrm{H}_{c}^{2 \mathrm{ht}(\nu)}\left(S_{\nu}, \quad\right) \cong \mathrm{H}_{S_{w_{0} \cdot \nu}}^{-2 \mathrm{ht}(\nu)}(\mathcal{G}, \quad)$.

Remark 4.10. The decomposition of $\mathcal{G}_{\lambda}$ into $N(\mathcal{K})$-orbits and $\bar{N}(\mathcal{K})$-orbits is an example of a perverse cell complex. Perverse cell complexes are the analogues of CW-complexes for computing cohomology of perverse sheaves instead of the 
ordinary cohomology. In the case at hand we are in the situation analogous to the one for $\mathrm{CW}$-complexes where the dimensions of all cells are of the same parity.

Remark 4.11. Theorem 2.7 follows from the estimate:

$$
\operatorname{dim}\left[m^{-1}(\nu) \cap\left(p^{-1}\left(\mathcal{G}_{\lambda}\right) \underset{G(\mathcal{O})}{\times} \mathcal{G}_{\mu}\right)\right] \leq \operatorname{ht}(\lambda+\mu+\nu),
$$

for coweights $\lambda, \mu, \nu \in X_{*}(T)$ such that $\lambda$ and $\mu$ are dominant and $\nu \in \overline{\mathcal{G}_{\lambda+\mu}}$. This claim follows from the estimate below which is a formal consequence of (4.6):

$$
\begin{aligned}
& \text { for a } T \text {-invariant subvariety } Y \subseteq \overline{\mathcal{G}_{\lambda}}, \\
& \qquad \operatorname{dim}(Y) \leq \max _{\nu \in \bar{Y} \cap X_{*}(T)} \operatorname{ht}(\lambda+\nu) .
\end{aligned}
$$

\section{The dual group}

We will now apply Tannakian formalism as in $[\mathrm{DM}]$ to $\mathrm{P}_{G(\mathcal{O})}(\mathcal{G}, \mathbb{k})$ and the functor $\mathbb{H}^{*}$. In sections 2 and 3 we have given a tensor product structure on the category $\mathrm{P}_{G(\mathcal{O})}(\mathcal{G}, \mathbb{k})$ via convolution and we have given functorial associativity and commutativity constraints for this tensor product. To see that $\mathrm{P}_{G(\mathcal{O})}(\mathcal{G}, \mathbb{k})$ is a rigid tensor category, we still must exhibit the identity object and construct duals. The identity object is given by the sky scraper sheaf supported on the point $1 \cdot G(\mathcal{O}) \in \mathcal{G}$ whose stalk is $\mathbb{k}$. The dual $A^{\vee}$ of a sheaf $A \in \mathrm{P}_{G(\mathcal{O})}(\mathcal{G}, \mathbb{k})$ is given as follows. Consider the following sequence of maps

$$
\mathcal{G} \stackrel{\pi}{\stackrel{\pi}{(}} G(\mathcal{K}) \stackrel{i}{\longrightarrow} G(\mathcal{K}) \stackrel{\pi}{\longrightarrow} \mathcal{G},
$$

where $i$ is the inversion on $G(\mathcal{K})$, i.e., $i(g)=g^{-1}$. We define an equivalence

$$
\iota: \mathrm{P}_{G(\mathcal{O})}(\mathcal{G}, \mathbb{k}) \rightarrow \mathrm{P}_{G(\mathcal{O})}(\mathcal{G}, \mathbb{k}) \text { by } \iota(A)=\pi_{*} \tilde{A} \text { where } i^{*} \tilde{A}=\pi^{*} A
$$

Then the dual $A^{\vee}$ is given by $A^{\vee}=\iota(\mathbb{D} A)$, where $\mathbb{D}$ denotes the Verdier dual.

In 4.3 we showed that $\mathbb{H}^{*}: \mathrm{P}_{G(\mathcal{O})}(\mathcal{G}, \mathbb{k}) \rightarrow \operatorname{Vec}_{\mathbb{k}}$ is a tensor functor. Corollary 4.5 says that $\mathbb{H}^{*}$ is exact and from this it is not hard to deduce that it is also faithful. Thus, we have verified that $\mathrm{P}_{G(\mathcal{O})}(\mathcal{G}, \mathbb{k})$ together with $\mathbb{H}^{*}$ constitutes a neutral Tannakian category and by [DM, theorem 2.11] we conclude:

Proposition 5.1. There exists an affine group scheme $\check{G}$ such that the tensor category $\mathrm{P}_{G(\mathcal{O})}(\mathcal{G}, \mathbb{k})$ is equivalent to the (tensor category) of representations of $\check{G}$. This equivalence is given via the fiber functor $\mathbb{H}^{*}$.

This result, as well as the result below, can also be found in [Gi]. We claim: 
Proposition 5.2. The affine group scheme $\check{G}$ is isomorphic to the Langlands dual of $G$.

To see this, one may argue as follows. First of all, as in [Gi] one easily finds that $\check{G}$ is noetherian and connected. By theorem 4.4b) we conclude that the dual torus $\check{T}$ of $T$ is contained in $\check{G}$ and then one shows, as in [Gi], that the torus $\check{T}$ is maximal. As one can express the root datum of a reductive group in terms of its irreducible representations one concludes, following [Gi], that the maximal reductive quotient of $\breve{G}$ is the dual group of $G$. It remains to eliminate the unipotent radical of $\check{G}$, but one can explicitly construct the group algebra of $\check{G}$ and it turns out to be of the same size as the the group algebra of the dual group. have certain non-trivial self extensions of objects and this can easily be ruled out (this argument is due to Soergel).

A few remarks are in order. Because $\check{G}$ is reductive, one concludes immediately that $\mathrm{P}_{G(\mathcal{O})}(\mathcal{G}, \mathbb{k})$ is semisimple. One can also see directly that $\mathrm{P}_{G(\mathcal{O})}(\mathcal{G}, \mathbb{k}) \cong \mathrm{P}_{\mathcal{S}}(\mathcal{G}, \mathbb{k})$ is semisimple, for example from [Lu, theorem 11c].

Let us make the statements of propositions 5.1 and 5.2 more concrete. Let $\lambda \in X_{*}(T) / W=X^{*}(\check{T}) / W$. To $\lambda$ we can associate an irreducible representation $V_{\lambda}$ of the Langlands dual group $\check{G}$ on one hand, and a $G(\mathcal{O})$-orbit $\mathcal{G}_{\lambda}$, and thus an irreducible perverse sheaf $\mathcal{V}_{\lambda}=j_{! *} \mathbb{k}_{\lambda}\left[\operatorname{dim} \mathcal{G}_{\lambda}\right], j: \mathcal{G}_{\lambda} \hookrightarrow \mathcal{G}$, on the other. Under the equivalence of proposition 5.1 the sheaf $\mathcal{V}_{\lambda}$ and the representation $V_{\lambda}$ correspond to each other. Furthermore, the representation space of $V_{\lambda}$ gets identified with the global cohomology of $\mathcal{V}_{\lambda}$, i.e., $V_{\lambda}=\mathbb{H}^{*}\left(\mathcal{G}, \mathcal{V}_{\lambda}\right)$. This interpretation gives a canonical basis for $\mathcal{V}_{\lambda}$ as follows. From theorem 4.4, the fact that $j_{! *} \mathbb{k}_{\lambda}\left[\operatorname{dim} \mathcal{G}_{\lambda}\right]={ }^{p} j_{!} \mathbb{k}_{\lambda}\left[\operatorname{dim} \mathcal{G}_{\lambda}\right]$, and $(4.6 \mathrm{c})$ we conclude:

$$
\begin{aligned}
\mathbb{H}^{k}\left(\mathcal{G}, \mathcal{V}_{\lambda}\right) & =\bigoplus_{\substack{\nu \in X_{*}(T) \\
k=2 \mathrm{ht}(\nu)}} \mathrm{H}_{c}^{2 \mathrm{ht}(\nu)}\left(S_{\nu}, \mathcal{V}_{\lambda}\right) \\
& =\bigoplus_{\substack{\nu \in X_{*}(T) \\
k=2 \operatorname{ht}(\nu)}} \mathrm{H}_{c}^{2 \mathrm{ht}(\lambda+\nu)}\left(S_{\nu} \cap \mathcal{G}_{\lambda}, \mathbb{k}\right) \\
& =\bigoplus_{\substack{\nu \in X_{*}(T) \\
k=2 \mathrm{ht}(\nu)}} \mathbb{k}\left[\operatorname{Irr}\left(S_{\nu} \cap \mathcal{G}_{\lambda}\right)\right]
\end{aligned}
$$

Here $\mathbb{k}\left[\operatorname{Irr}\left(S_{\nu} \cap \mathcal{G}_{\lambda}\right)\right]$ denotes the vector space spanned by the irreducible components of $S_{\nu} \cap \mathcal{G}_{\lambda}$. Thus we get

$$
V_{\lambda}=\mathbb{H}^{*}\left(\mathcal{G}, \mathcal{V}_{\lambda}\right)=\bigoplus_{\nu \in X_{*}(T)} \mathbb{k}\left[\operatorname{Irr}\left(S_{\nu} \cap \mathcal{G}_{\lambda}\right)\right]
$$

Note that the results above imply that the cohomology group $\mathbb{H}^{*}\left(\mathcal{G}, \mathcal{V}_{\lambda}\right)$ is generated by algebraic cycles. 


\section{The case of rings and arbitrary fields}

In this section we briefly indicate how the results in this paper can be extended to the case of rings. To this end, let $\mathbb{k}$ be a commutative, unital, Noetherian ring of finite global dimension. We write $\mathrm{P}_{G(\mathcal{O})}(\mathcal{G}, \mathbb{k})$ for the category of $G(\mathcal{O})$ equivariant perverse sheaves on $\mathcal{G}$ with coefficients in $\mathbb{k}$. Recall that this means, in particular, that the stalks of the perverse sheaves are finitely generated $\mathbb{k}$ modules. All the results in sections 2-4 go through in this context except that in order that the convolution of two perverse sheaves be perverse one needs one of the sheaves to be in the full subcatgeory $\mathrm{P}_{G(\mathcal{O})}^{\text {proj }}(\mathcal{G}, \mathbb{k})$ consisting of sheaves $\mathcal{F}$ such that $\mathbb{H}^{*}(\mathcal{G}, \mathcal{F})$ is projective over $\mathbb{k}$. The Tannakian formalism yields

6.1 Proposition. There exists an affine group scheme $\check{G}$ over $\operatorname{Spec}(\mathbb{k})$ such that the tensor category $\mathrm{P}_{G(\mathcal{O})}^{\text {proj }}(\mathcal{G}, \mathbb{k})$ is equivalent to the tensor category of representations of $\breve{G}$ which are projective as $\mathbb{k}$-modules.

Let us write ${ }^{L} G$ for the unique split reductive group scheme over $\operatorname{Spec}(\mathbb{Z})$ corresponding to the root datum dual to that of $G$ and let ${ }^{L} G_{\mathbb{k}}$ denote the corresponding group scheme over $\operatorname{Spec}(\mathbb{k})$. Then

6.2 Theorem. The group scheme $\check{G}$ is isomorphic to ${ }^{L} G_{\mathbb{k}}$.

The main new point is to show that $\check{G}$ is reduced, this is not a priori clear when $\mathbb{k}$ is not a field of characteristic zero. We analyze explicitly the proprojective object which represents the functor $\mathbb{H}^{*}: \mathrm{P}_{G(\mathcal{O})}(\mathcal{G}, \mathbb{k}) \rightarrow\{\mathbb{k}$-modules $\}$. Its Verdier dual corresponds to the group algebra of ${ }^{L} G_{\mathbb{k}}$. With the help of the projective one finds that the equivalence (6.1) canonically extends to an equivalence of abelian categories $\mathrm{P}_{G(\mathcal{O})}(\mathcal{G}, \mathbb{k})$ and the category of representations of $\check{G}$. The proof that $\check{G}$ is integral (hence reduced), is based on the perverse-sheaf analogue of the fact that the tensor product of Weyl modules has a filtration such that the succesive subquotients are Weyl modules.

Each $G(\mathcal{O})$-orbit $\mathcal{G}_{\lambda} \stackrel{j}{\hookrightarrow} \mathcal{G}$, defines two standard perverse sheaves $\mathcal{I}_{\lambda}^{!}(\mathbb{k}) \stackrel{\text { def }}{=}{ }^{p} \mathrm{H}^{0}\left(R j_{!} \mathbb{k}_{\mathcal{G}_{\lambda}}\left[\operatorname{dim}\left(\mathcal{G}_{\lambda}\right)\right]\right)$ and $\mathcal{I}_{\lambda}^{*}(\mathbb{k}) \stackrel{\text { def }}{=}{ }^{p} \mathrm{H}^{0}\left(R j_{*} \mathbb{k}_{\mathcal{G}_{\lambda}}\left[\operatorname{dim}\left(\mathcal{G}_{\lambda}\right)\right]\right)$. The usual (middle perversity) intersection homology sheaf $\mathcal{V}_{\lambda}(\mathbb{k}) \stackrel{\text { def }}{=} j_{\text {!* }} \mathbb{k}_{\mathcal{G}_{\lambda}}\left[\operatorname{dim}\left(\mathcal{G}_{\lambda}\right)\right]$, is the image of the canonical map $\mathcal{I}_{\lambda}^{!}(\mathbb{k}) \rightarrow \mathcal{I}_{\lambda}^{*}(\mathbb{k})$. (All three coincide if $\mathbb{k}$ is a field of characteristic zero.) These standard perverse sheaves are topological realizations of the Weyl module $\mathbb{H}^{*}\left(\mathcal{G}, \mathcal{I}_{\lambda}^{!}(\mathbb{k})\right)$ and its dual $\mathbb{H}^{*}\left(\mathcal{G}, \mathcal{I}_{\lambda}^{*}(\mathbb{k})\right)=$ sections of a line bundle on the flag variety. The arguments in $\S 5$ now yield canonical bases of both the Weyl module and its dual. (When $\mathbb{k}$ is a field of characteristic zero these are two bases of $\mathbb{H}^{*}\left(\mathcal{G}, \mathcal{V}_{\lambda}(\mathbb{k})\right)$.) We conclude with the following

6.3 Conjecture. The stalks of $\mathcal{I}_{\lambda}^{!}(\mathbb{Z})$ and the costalks of $\mathcal{I}_{\lambda}^{*}(\mathbb{Z})$ have no torsion.

\section{The Satake isomorphism}

In this section we briefly discuss the case of the classical Satake isomorphism. Let $G$ be a split reductive group over $\mathbb{F}_{q}$. The construction of the affine Grass- 
mannian can be performed in this context exactly the same way as over $\mathbb{C}$, with $\mathcal{O}=\mathbb{F}_{q}[[t]]$ and $\mathcal{K}=\mathbb{F}_{q}((t))$. We consider the category $\mathrm{P}_{G(\mathcal{O})}\left(\mathcal{G}, \overline{\mathbb{Q}_{\ell}}\right)$ of $G(\mathcal{O})$-equivariant pure perverse $\overline{\mathbb{Q}}$-sheaves of weight zero on $\mathcal{G}$. The convolution product preserves this category and the results in sections 2-5 go through in this context, resulting in the following:

7.1 Theorem. There is an equivalence of tensor categories

$$
\mathrm{P}_{G(\mathcal{O})}\left(\mathcal{G}, \overline{\mathbb{Q}_{\ell}}\right) \cong \operatorname{Rep}\left({ }^{L} G_{\overline{\mathbb{Q}_{\ell}}}\right) .
$$

Let us now pass to the Grothendieck groups on both sides of 7.1. On the right hand side we get the representation $\operatorname{ring} \operatorname{Rep}\left[{ }^{L} G_{\overline{\mathbb{Q}_{\ell}}}\right]$. It remains to interpret the Grothendick group of the left hand side. Because we are considering the category of pure sheaves only, passing from sheaves to functions on $\mathcal{G}\left(\mathbb{F}_{q}\right)$ via the trace of Frobenius results in an isomophism of the Groethendieck group of $\mathrm{P}_{G(\mathcal{O})}\left(\mathcal{G}, \overline{\mathbb{Q}_{\ell}}\right)$ and the spherical Hecke algebra $\mathcal{H}$ of compactly supported $G\left(\mathbb{F}_{q}[[t]]\right)$-bi-invariant functions on $G\left(\mathbb{F}_{q}((t))\right)$. Thus theorem 7.1 yields an isomorphism of $\overline{\mathbb{Q}_{\ell}}$-algebras

$$
\mathcal{H} \cong \operatorname{Rep}\left[{ }^{L} G_{\overline{\mathbb{Q}_{\ell}}}\right] .
$$

This is the classical Satake isomorphism.

7.3 Remark. Some of the arguments for sections 2-5 have to modified slightly when working in the context of $\ell$-adic sheaves. This has been done, in a way different from ours, earlier by Ngo in $[\mathrm{N}]$.

\section{References}

[BBD] A. Beilinson, J. Bernstein and P. Deligne, Faisceaux pervers, Analysis and topology on singular spaces, I (Luminy, 1981), Astérisque, 100, Soc. Math. France, Paris, 1982, pp. $5-171$.

[BL1] A. Beauville and Y. Laszlo, Conformal blocks and generalized theta functions, Comm. Math. Phys. 164 (1994), 385-419.

[BL2] _ Un lemme de descente, C. R. Acad. Sci. Paris Sér. I Math. 320 (1995), 335340.

[DM] P. Deligne and J. Milne, Tannakian categories, Hodge cycles and motives, Lecture Notes in Mathematics, 900, Springer-Verlag, Berlin-New York, 1982, pp. 101-228.

[Gi] V. Ginzburg, Perverse sheaves on a loop group and Langlands duality, preprint (1995), alg-geom/9511007.

[LS] Y. Laszlo and C. Sorger, The line bundles on the stack of parabolic G-bundles over curves and their sections, preprint (1996), alg-geom/9507002.

[Lu] G. Lusztig, Singularities, character formulas, and a q-analogue for weight multiplicities, Analyse et topologie sur les espaces singuliers, Astérisque, 101-102, Soc. Math. France, Paris, 1982, pp. 208-229.

[N] Ngô Báo Châu, Le lemme fondamental de Jacquet et Ye en caractristique positive, Duke Math. J. 96 (1999), 473-520. USA

Department of mathematics, University of Massachusetts, Amherst, MA 01002,

E-mail address: mirkovic@math.umass.edu

Department of mathematics, Brandeis University, Waltham, MA 02454, USA

E-mail address: vilonen@math.brandeis.edu 